\title{
Lymphomatoide Kontaktdermatitis im rotfarbenen Anteil einer mehrfarbigen Tätowierung bei einem 21-jährigen Patienten
}

\author{
Lymphocytic Contact Dermatitis Developing in the Red Dyed Part \\ of a Multi-Coloured Tattoo of a 21-Year-Old Patient
}

Autoren

Institute
S. Vandersee' ${ }^{1}$, R. Ulrich², H.-J. Günzl ${ }^{3}$, S. Merkelbach-Bruse ${ }^{4}$, F. Fronhoffs ${ }^{4}$, R. Hartmann ${ }^{1}$

Bundeswehrkrankenhaus Berlin, Abteilung für Dermatologie

Bundeswehrkrankenhaus Hamburg, Abteilung für Dermatologie

Praxis für Dermatologie, Dermatohistopathologie, Hamburg

${ }^{4}$ Institut für Pathologie, Universitätsklinikum Bonn

\section{Bibliografie}

DOI http://dx.doi.org/

10.1055/s-0029-1243905

Online-Publikation: 11. 2. 2010

Akt Dermatol 2010; 36:

232-234 @ Georg Thieme

Verlag KG Stuttgart · New York ISSN 0340-2541

Korrespondenzadresse Dr. med. Staffan Vandersee Bundeswehrkrankenhaus Berlin Abteilung Dermatologie, Venerologie, Allergologie Scharnhorststraße 13

10115 Berlin

svandersee@gmx.de

\section{Zusammenfassung}

$\nabla$

Kutane lymphomatoide Infiltrationen auf dem Boden einer Tätowierung sind eine seltene Komplikation auf das Einbringen des Pigments (insbesondere rotes). Weitaus häufiger sind tätowierungsinduzierte chronische Kontaktdermatitiden. Da pseudolymphomatoide Kontaktreaktio-

\section{Einleitung}

$\nabla$

Kontaktreaktionen auf Tätowierungen sind insgesamt seltene Ereignisse, wegen der Vielzahl tätowierter Individuen aber dennoch häufiger zu beobachten. Meistens handelt es sich um Kontaktdermatitiden bei Unverträglichkeit der roten Farbstoffe, die häufig Quecksilbersalze enthalten. Selten wurden jedoch auch pseudolymphomatoide Unverträglichkeitsreaktionen beschrieben. Wir berichten über den Fall eines 21-jährigen Patienten, bei dem sich nach der Teilexzision eines therapierefraktär ekzematoiden Tätowierungsteils die histologische Diagnose einer lymphomatoiden Kontaktdermatitis ergab. Vorgestellt werden zudem die Ergebnisse der durchgeführten molekularbiologischen und immunhistochemischen Diagnostik.

\section{Kasuistik \\ $\nabla$}

\section{Anamnese}

Im Februar 2009 stellte sich der 21-jährige männliche Patient erstmalig in unserer Abteilung ambulant vor. Er hatte sich im Frühjahr 2006 durch ein Tätowierungsstudio eine zweifarbige (rot und blau) Tätowierung am rechten Unterarm anfertigen lassen. Nach initialer Beschwerdefreiheit war es im Sommer 2008, streng begrenzt auf den rot gefärbten Anteil, zu einer chronischen, therapierefraktären und stark juckenden Ekzematisation nen rare Ereignisse sind und die Prognose unsicher ist, wird in der Regel zur Exzision geraten. Wir berichten über den Fall eines 21-jährigen Patienten, bei dem sich die im rotfarbenen Anteil einer zweifarbigen Tätowierung befindlichen Ekzematisationen nach erfolgter Exzision als lymphomatoide Kontaktdermatitis erwiesen.
Aufnahmebefund

Es imponierte im Bereich einer den gesamten Unterarm beugeseitig bedeckenden, zweifarbigen, roten und blauen Tätowierung eine streng auf den Bereich des roten Pigments limitierte Rötung mit deutlicher Infiltration und lokalisierter Vesikelbildung. Der übrige Haut- und Schleimhautbefund zeigte sich unauffällig ( $\bullet$ Abb. 1). 


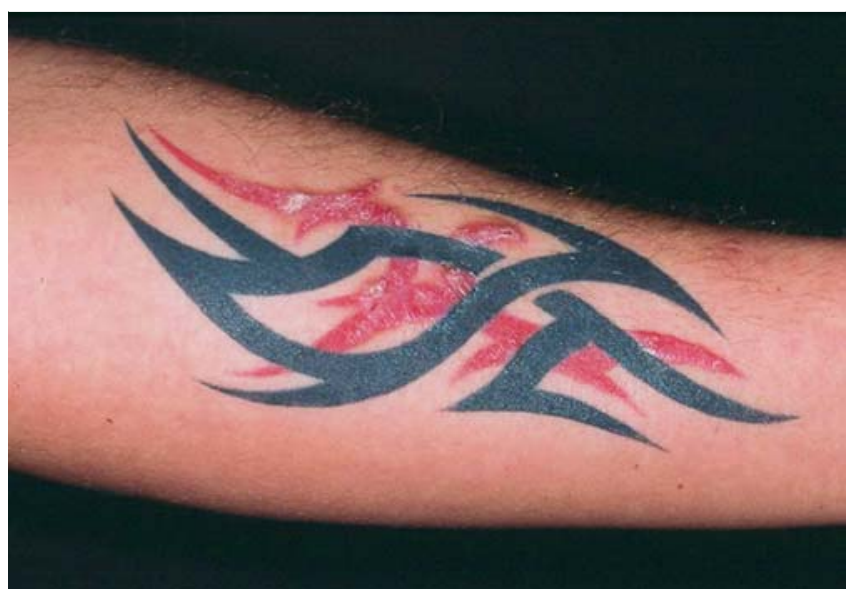

Abb. 1 Makroskopische Aufnahme des Unterarms vor Therapiebeginn.

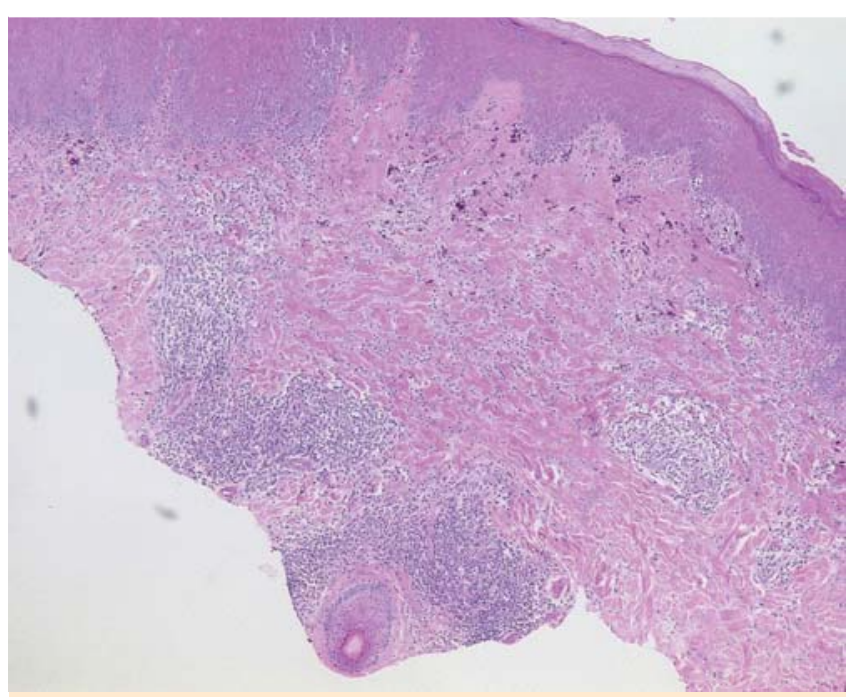

Abb. 2 Histologische Übersichtsaufnahme des Resektats (HE, $5 \times$ Vergrößerung).

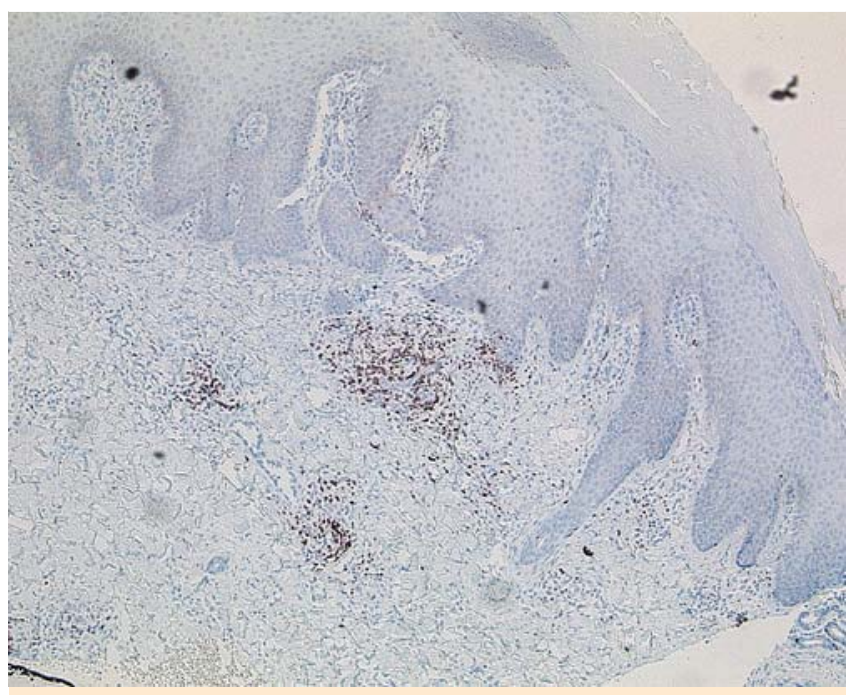

Abb. 3 Immunhistochemische Resektatfärbung mit anti-CD-3 (5 × Vergrößerung).

\section{Labor}

Das Aufnahmelabor zeigte keine Auffälligkeiten, insbesondere im Hinblick auf das Differenzialblutbild.

\section{Histologie}

Die histologische Aufarbeitung ergab eine chronische, ekzematoide Dermatitis mit pseudolymphomartiger Fremdkörperreaktion und Einschluss eines roten Pigments. Obwohl ein Lymphom im exzidierten Material nicht feststellbar war, wurden weitergehende molekularbiologische und immunhistochemische Untersuchungen durchgeführt. Es zeigte sich eine polyklonale T-ZellExpansion mit einem gemischtzelligen Immunphänotyp von CD-4- wie auch CD-8-Lymphozyten sowie begleitenden Plasmazellen ( $\bullet$ Abb. 2 u. 3). Aufgrund dieser histologischen Charakterisierung wurde die Diagnose einer lymphomatoiden Kontaktdermatitis gestellt.

\section{Therapie und Verlauf \\ $\nabla$}

Nach Diskussion der verfügbaren therapeutischen Modalitäten entschloss sich der Patient zu einer Teilexzision der Ekzematisation. Je nach Wundheilungsergebnis würden dann in der Folge weitere Serienexzisionen erfolgen. Der erste Eingriff wurde am 11.3. 2009 durchgeführt. Es wurde ein ca. $10 \times 3 \mathrm{~cm}$ durchmessendes keilförmiges Hautareal exzidiert und der Defekt durch eine lokale Verschiebelappenplastik gedeckt. Der Unterarm wurde durch eine Gipslonguette ruhiggestellt, der Fadenzug erfolgte am 14. postoperativen Tag. Wegen der überraschenden Diagnose einer lymphomatoiden Kontaktdermatitis und der einhergehenden unsicheren Prognose wurde dem Patienten zu weiteren Serienexzisionen geraten, er wurde am 25.3.2009 aus der stationären Behandlung entlassen, die Narbe mit Silikongel nachbehandelt und Schonung des rechten Arms empfohlen.

Bei der nächsten stationären Aufnahme am 8.6.2009 zeigte sich im Bereich der Exzisionsnarbe eine Hypertrophie mit einer Verbreiterung auf $1 \mathrm{~cm}$ sowie einigen punktförmigen Ulzera. Entnommene Probeexzisionen aus der Narbe konnten kein lymphoides Infiltrat mehr nachweisen, solche aus den ekzematösen Arealen zeigten erneut den Befund eines Pseudolymphoms. Postoperativ kam es zu einer Wundinfektion, die antibiotisch behandelt wurde. Nach der Entlassung des Patienten wurde er im August 2009 letztmalig aufgenommen und alle verbliebenen roten Tätowierungsanteile operativ entfernt.

\section{Diskussion}

$\nabla$

Kontaktdermatitiden nach Tätowierungen sind eingedenk der hohen Frequenz tätowierter Individuen insgesamt selten, für rote Farbstoffe allerdings häufiger beschrieben als für andere Färbungen [1]. In jüngerer Zeit haben Azofarbstoffe [2] die klassischen Schwermetallpigmente (bei Rot insbesondere Quecksilber und Cadmium sowie Titandioxid als Füllmittel) [3] verdrängt. In der Regel ist, obwohl eine Allergenelimination nicht stattfindet, eine topische Glukokortikoidtherapie oft ausreichend wirksam und bewirkt nachhaltige Remissionen. Pseudolymphomatoide Kontaktreaktionen nach einer Tätowierung sind in der Literatur weitaus seltener beschrieben (8 Fallberichte in pubmed seit 2000). Hierbei ist zu überlegen, ob die Erkrankung nicht eher selten diagnostiziert als tatsächlich selten ist, da in der Annahme, dass eine Kontaktdermatitis vorliegt, Probeexzisionen eher unterbleiben. 


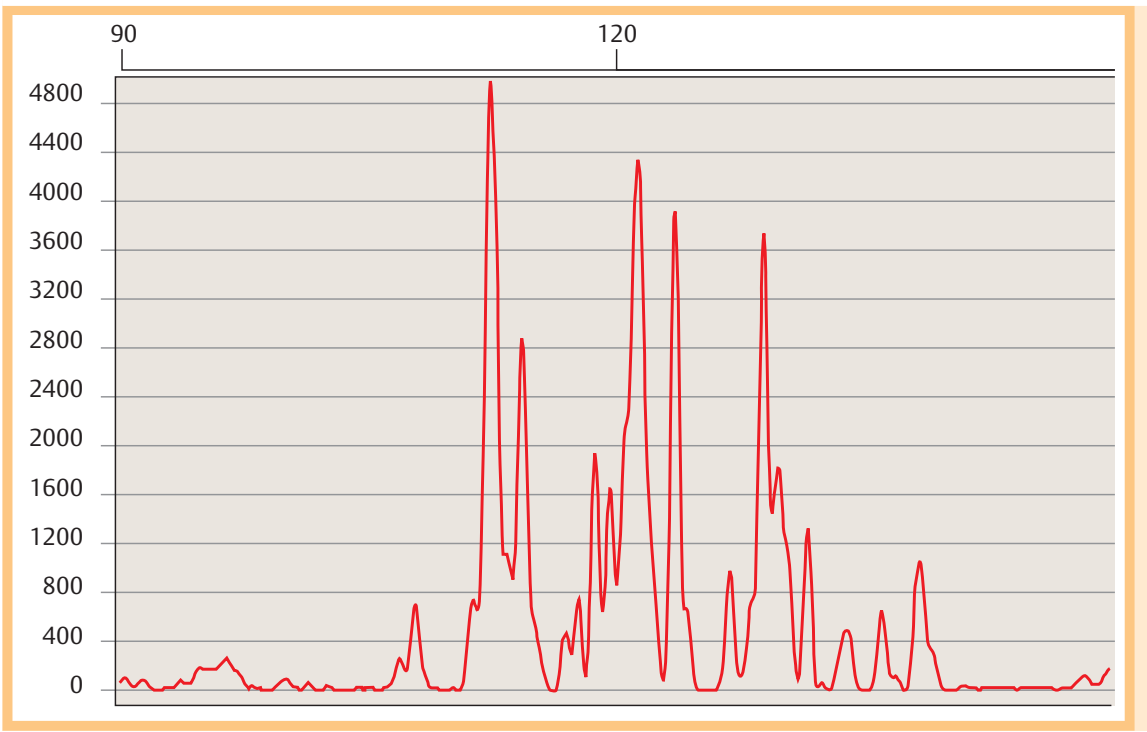

Abb. 4 Elektropherografische Darstellung der $\mathrm{Y}$-T-Zell-Rezeptor-DNA-Amplifikation.
Definiert sind kutane lymphoide Hyperplasien als gutartige, hyperplastische Hautinfiltrate, die klinisch und histologisch Lymphome imitieren [4]. Seit der Einführung der Klonalitätsdiagnostik für IgH (bei B-Zellen) und die T-Zell-Rezeptor-DNA (bei T-Zellen) ist bekannt, dass sich in der Mehrzahl der Fälle polyklonale Zell-Expansionen nachweisen lassen ( $\bullet$ Abb.4). Dies ist in der Abgrenzung zu malignen Lymphomen entscheidend. In der Mehrzahl der Fallberichte lagen T-Zell-Infiltrate vor, B-Zellen waren seltener beteiligt [5]. Diskutiert wird ferner, ob ein protrahiertes Verbleiben des Pigments in situ im Verlauf zu einer malignen Transformation der Zellen führt. Wegen der geringen Fallzahl ist dies nicht eindeutig zu beantworten, zumal beobachtendes Zuwarten von keinem der Autoren als therapeutische Option gewählt wurde. Es existiert in der Literatur lediglich ein Bericht eines malignen Lymphoms auf dem Boden einer Tätowierung [6]. Aufgrund dieser unsicheren Prognose ist in fast allen berichteten Fällen die Exzision erfolgt. Eine aktuelle Arbeit beschreibt jedoch den erfolgreichen Therapieversuch mit Hydroxychloroquin [7].

\section{Fazit}

$\nabla$

In jedem Fall sollte bei therapierefraktären Kontaktdermatitiden nach erfolgter Tätowierung an eine lymphoide Hyperplasie gedacht und eine Probeexzision erwogen werden. Bestätigt sich der Verdacht, ist die Exzision nach heutigem Kenntnisstand die sicherste Option, um Spätentartungen vorzubeugen.

\section{Abstract}

\section{Lymphocytic Contact Dermatitis Developing in the Red Dyed Part of a Multi-Coloured Tattoo of a 21-Year-Old Patient $\nabla$}

Lymphomatoid contact dermatitis is a rare complication after tattooing, mostly occurring when red dye is used. More common is tattoo-induced chronic contact dermatitis. Pseudolymphomalike contact-reactions are uncommon and the prognosis is to be considered unclear. Therefore excision is the most widely recommended treatment. We report the case of a 21-year-old patient, whose therapy-resistant contact dermatitis in the area of a red dyed tattoo proved to be a lymphocytic contact dermatitis.

\section{Literatur}

1 Mortimer NJ, Chave TA, Johnston GA. Red tattoo reactions. Clin Exp Dermatol 2003; 28: $508-510$

2 Engel E, Santarelli F, Vasold R, Maisch T, Ulrich $H$ et al. Modern tattoos cause high concentrations of hazardous pigments in skin. Contact Dermatitis 2008; 58: 228-233

3 Lehmann G, Pierchalla P. Tätowierungsfarbstoffe. Dermatosen 1988; 36: $152-156$

4 Gilliam AC, Wood GS. Cutaneous lymphoid hyperplasias. Seminars in Cutaneous Medicine and Surgery 2000; 19: 133-141

5 Riljardsdam JU, Bruynzeel DP, Vos W, Meijer CJ, Willemze R. Immunhistochemical studies of lymphadenosis benigna cutis occurring in a tattoo. Am J Dermatopathol 1988; 10: 518-523

6 Armiger WG, Caldwell EH. Primary lesion of a non-Hodgkin's lymphoma occurring in a skin tattoo: case report. Plast Reconstr Surg 1978; 62: $125-127$

7 Patrizi A, Raone B, Savoia F, Bacci F, Pileri A et al. Tattoo-associated pseudolymphomatous reaction and its successful treatment with hydroxychloroquine. Acta Derm Venereol 2009; 89: 327-328 